\title{
Higher Incidence of Enterohemorrhagic Escherichia coli (EHEC) in Autistic Children and Their Potential Role in Exacerbation of Autistic Enterocolitis
}

\author{
Joby Pulikkan \\ Department of Genomic Science, School of Biological Sciences, Central University of Kerala, \\ Padannakad, Kasaragod, Kerala, India \\ E-mai: puljobcmi@gmail.com
}

Rajendra Kumar Mullur Govindan Nadar

Department of Genomic Science, School of Biological Sciences, Central University of Kerala, Padannakad, Kasaragod, Kerala, India

E-mail: rajum8820@gmail.com

\section{Binoop Mohan}

Department of Genomic Science, School of Biological Sciences, Central University of Kerala, Padannakad, Kasaragod, Kerala, India

E-mail: binoopm28291@gmail.com

Amitha Chirayath Pallipuram Joy

Department of Biotechnology Engineering, Sahrdaya College of Engineering \& Technology,

Kodakara, Thrissur, Kerala, India

E-mail: amithajoy2009@gmail.com

Tony Grace (Corresponding author)

Department of Genomic Science, School of Biological Sciences, Central University of Kerala, Padannakad, Kasaragod, Kerala, India

E-mail: tonygrace99@gmail.com 
Received: May 4, 2016 Accepted: June 30, 2016 Published: July 3, 2016

Doi: 10.5296/jab.v4i2.9417ＵRL: http://dx.doi.org/10.5296/ jab.v4i2.9417

\begin{abstract}
Based on the hypothesis that abnormal pathogens in the bowel can affect the brain and consequently can play a major role in the exacerbation of autistic symptoms, we have been in an attempt to detect and identify intimin gene producing microorganism Escherichia coli from stools of autistic children using culture-independent techniques. The presence of intimin gene was detected using polymerase chain reaction (PCR) with specific primers. Sequence of the gene amplified after PCR matched to the sequence of intimin gene with $100 \%$ accuracy. EHEC strains are found to produce strong cytotoxins, the important virulence attribute of EHEC being the intimin protein encoded by eaeA gene. Hitherto deemphasized and played down its role in autistic children, the intimin gene was found in a significant number in the stools of children with autism compared to normal children. Our results, thus, show a high degree of prevalence of eaeA positive $E$. Coli in children with autism. A relevant question is if this greater prevalence of EHEC can be causative to the digestion problems observed in autism which may further affect brain and cognitive functions. In order to understand the role of intimin as a prospective candidate leading to cognitive dysfunctions, we had an in silico study on the effects of intimin on selected neurotransmitters which also showed a profound significance with all the docked conformations giving negative binding energy in the order serotonin, dopamine and acetyl choline.
\end{abstract}

Keywords: Autism, EHEC, Intimin, Autistic enterocolitis, Diarrhea, Autodock, Docking

\title{
1. Introduction
}

Autism spectrum disorders (ASD) are pervasive neurodevelopment disorders known to have impaired communication, social abnormalities and stereotyped behavioral patterns as its characteristic features (Williams et al., 2012; D'Souza et al., 2006). In addition to heritable predisposition to the diseases, environmental factors are also suggested to play a major role in autism (Finegold et al., 2010). The anecdotal reports as well as several studies claim that children with autism have frequent gastrointestinal problems and most of them suffer from bloating, abdominal pain, constipation, diarrhea, indigestion and more (Benach et al., 2012; McDermott et al., 2006). Often irritability, aggressive behavior, mood changes, discomfort, and nightmare awakenings found in autistic population are misinterpreted as neurological or behavioral problems (Jepson \& Johnson, 2007) where in veracity it could be due to the related GI symptoms. In this scenario, it is interesting to note that a new variety of inflammatory bowel disease known as autistic enterocolitis has been put forth by Wakefield and colleagues which is defined by chronic patchy inflammation and lymphonodular hyperplasia (LNH) as its characteristics (Jepson \& Johnson, 2007). They also have mentioned about the active neutrophilic inflammation in the ileum of autistic children (8\%) compared to none in the control. Neutrophils, the first line of defense against bacteria, are known to have the ability in killing bacteria (Jepson \& Johnson, 2007). This has initiated us to propose a study on the role of Enterohaemorragic $E$. coli as a prospective cause of autistic enterocolitis 
in a subtype of autism where diarrhea is observed as a GI disturbance.

Enterohaemorrhagic E. coli (EHEC) serotype O157:H7 is a human pathogen (Nguyen et al., 2012). Enterohaemorrhagic E.coli is an important cause of acute gastroenteritis in humans (Nataro \& Kaper, 1998), also being a causative agent for a wide spectrum of illnesses including mild diarrhoea and various diseases such as haemorrhagic colitis and haemolytic uraemic syndrome (HUS) (Mundy et al., 2007). EHEC are endowed with a histopathological feature known as attaching and effacing (A/E) lesions (Mundy et al., 2007) colonizing the colon and promoting electrolyte imbalance (Nguyen et al., 2012).One among the major components that can evoke A/E lesion, the outer membrane adhesion intimin (Fagan et al., 1999) is encoded mainly by the locus of enterocyte effacement (LEE) which is a chromosomal pathogenicity island (Blanco et al., 2006). Intimin is a key virulent factor implicated in the pathogenesis of enterohemorrhagic Escherechia coli (EHEC) (Cookson et al., 2003). Intimin is a 97-KDa attachment and effacement protein encoderd by eaeA (Jerse et al., 1990; McKee et al., 1996).

It is proved without doubt that the traditional culture-based methods fail to bring the optimum outcome with respect to profiling of bacteria resulting in a considerable underestimation of bacteria present in fecal samples (Gafan et al., 2005). Thus, molecular techniques introduced in microbial ecology have made a marked improvement in studying the composition of intestinal flora in a culture-independent way. Among various approaches, PCR analysis using specific primers brings about very sensitive results with an ease of use and speed (Wang et al., 1996) which is successfully furnished here. The novelty of the approach is that seldom before EHEC has been implicated in autistic enterocolitis. PCR analysis is successful in surpassing the limitations of culture techniques, which followed by an in silico analysis can prove it.

This is the backdrop which prompted us in undertaking a screening study for the identification of intimin producing eae A gene of EHEC (intimin here serves as a marker of EHEC) in autism using culture independent techniques. The aim of this study was to establish the role of EHEC in the diarrheagenic tendency involved in autism and their potential role in affecting selected neurotransmitters which are crucial in the normal functioning of cognitive system.

\section{Materials and Methods}

\subsection{Autistic Children}

25 autistic candidates and 25 healthy cohorts aged between 3 and 12 were admitted into the study with their informed parental consent. The purpose of the experiment was carefully explained to the parents and the caretakers of the children. Clinical and historical examination data of the children involved in the study were collected along with parental consent forms. Children with minimum score of thirty in a CARS rating were included in the study. CARS rating was carried out by experts in the field.

\subsection{Sample Collection and DNA Extraction}

Feces were collected in sterile containers, packed in ice packs in cooler box soon after defecation, and were transported to laboratory. Upon reaching the laboratory, total bacterial DNA was isolated from the stool sample using QIAamp DNA stool Kit as per the manufacturer's protocol. 


\subsection{Detection of Virulence eae A Gene}

DNA from stool samples were used for amplification, using specific primer eae $A$ forward primer (GTGGCGAATACTGGCGAGACT), and reverse primer (CCCCATTCTTTTTCACCGTCG) obtained from a previous study (Fagan et al, 1999). Amplification of bacterial DNA was performed with $2.5 \mu 1$ of bacterial DNA and $0.75 \mu 1$ of each primer in a $25 \mu \mathrm{l}$ of PCR reaction mixture. The PCR reaction mixture contained $1 \times$ of Promega master mix. The size of the expected amplified product was $890 \mathrm{bp}$. The PCR cycle included initial denaturation of $95{ }^{\circ} \mathrm{C}$ for 3 minutes followed by 35 cycles of $95{ }^{\circ} \mathrm{C}$ of 20 seconds for denaturation, $58{ }^{\circ} \mathrm{C}$ of 40 seconds for annealing, and $72{ }^{\circ} \mathrm{C}$ of 1.30 minutes for extension.

\subsection{DNA Sequencing of eae A Gene}

Sequencing was carried out using an automated ABI 3100 Genetic Analyser. Sequences obtained were compared with similar sequences of the reference organisms by BLAST search. Sequence data were aligned with Clustal W package.

\subsection{Statistical Analysis}

Statistical analysis was done using R Package (R Core Team 2007). Testing procedures to detect significant differences between specified groups was done using Student's t-test. Associations were considered statistically significant if the $p$ value was $<0.05$ using a 2-tailed test which could effectively suggest the association between gut/intestine problems and the presence of the intimin gene in a cohort including autistic children against normal.

\subsection{In silico Study on the Effects of Intimin on Selected Neurotransmitters}

\subsubsection{Ligand Preparation}

The chemical structures of serotonine, dopamine and acetylcholine were drawn in Advanced Chemistry Development Inc. (ACDLabs) Version 11 (www.acdlabs.com), Chemsketch. The structure was cleaned and energy optimized. The pdb file formats of the structures were obtained using iConfileformat converter (pipe@ibiosolutions.com).

\subsubsection{Protein Structure Prediction}

The obtained intimin sequence was translated using Translate tool of Expasy (Expasy Bioinformatics Resource portal, expasy.org/translate/). The three dimensional structure of the protein was obtained by homology modeling using Swissmodel server (Arnold et al., 2006). The templates were obtained using blastp (Altschul et al., 1990). The PDBids (Protein Data Bank) of the selected templates were 4E1S and 4E1T. The templates were superimposed and prepared for submission using spdbv (swisspdb viewer) (Nicolas et al., 2006). The 3D structure predicted was retrieved from Swissmodel server. The Ramachandran plot of the structure was also obtained. The modeled structure was used as the protein target for the docking studies.

\subsubsection{Docking}

Autodock 4.2 Linux version was used for docking (Morris et al., 2009). The protein target was loaded and polar hydrogen atoms were added. Ligand structures were also loaded and the torsions were chosen. For the ligands Gasteiger charges were added and nonpolar hydrogen atoms were merged. All the rotatable bonds were set to be rotatable. Grid preparation was 
done and the grid box with a dimension of $70 \times 65 \times 65$ points and $0.436 \AA$ grid spacing were used around each binding pocket.The grid parameter file specifies an AutoGrid calculation, counting the size and position of thegrid, the atom types that will be used, the coordinate file for the rigid receptor, and otherparameters for calculation of the grids (Garrett et al). Rigid docking was carried out using Lamarckian Genetic Algorithm. Autogrid file and Autodock docking parameter files were generated for all the three ligands. After docking searches were finished, the best conformation was selected from the most populated cluster with the least binding energy. The interaction of docked protein-ligand complex conformations, including hydrogen bond and other interactions were analyzed.

\section{Results}

\subsection{DNA Isolation}

Total bacterial DNA was isolated from the stool sample using QIAamp DNA stool Kit as per the manufacturer's protocol. Clear bands were obtained in gel electrophoresis using $1 \%$ agarose gel. All the samples gave a concentration of $30 \mathrm{ng}-100 \mathrm{ng}$ per microlitre with purity of 1.8. The DNA was stored in $-20^{\circ} \mathrm{C}$ for further analyses.

\subsection{Detection of Virulence eae A Gene}

DNA from stool samples were used for amplification using specific primer eae $A$ forward primer (GTGGCGAATACTGGCGAGACT), and reverse primer (CCCCATTCTTTTTCACCGTCG). The positive samples were identified which had 890 base pair product (Figure1). Eighteen out of twenty five test samples had been detected with eae $A$ gene while only 5 out of twenty five control samples were detected to have the particular gene of interest.

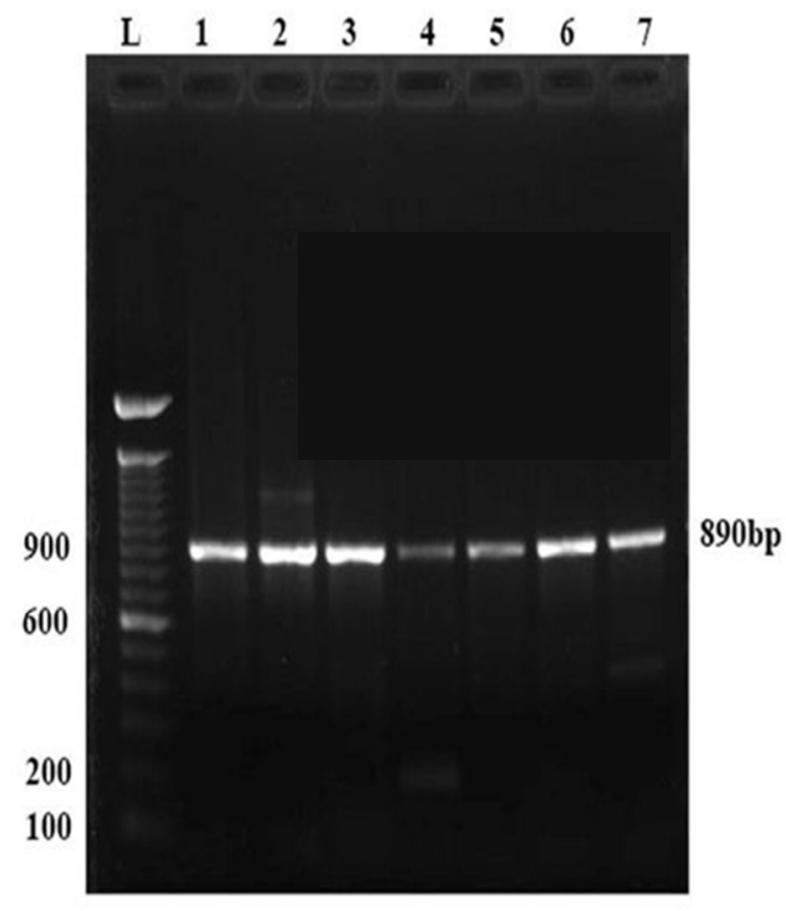

Figure 1. Amplified Virulence eae A gene 


\section{Macrothink}

\subsection{DNA Sequencing of eae A Gene}

The sequences obtained were compared with similar sequences of the reference organisms by BLAST search. $100 \%$ similarity was obtained which further confirm that the organism involved is EHEC.

\subsection{Statistical Analysis}

Presence of intimin gene and therefore EHEC occurred significantly more frequently in stool sample of Autistic children $(72 \%, 18 / 25)$ as compared to $(19 \%, 4 / 21)$ (Figure 2) normal children $(p=0.0009)$ with a standard error of 0.091766 and 0.105131 respectively.

Statistical Comparison of EaeA in Normal and ASD

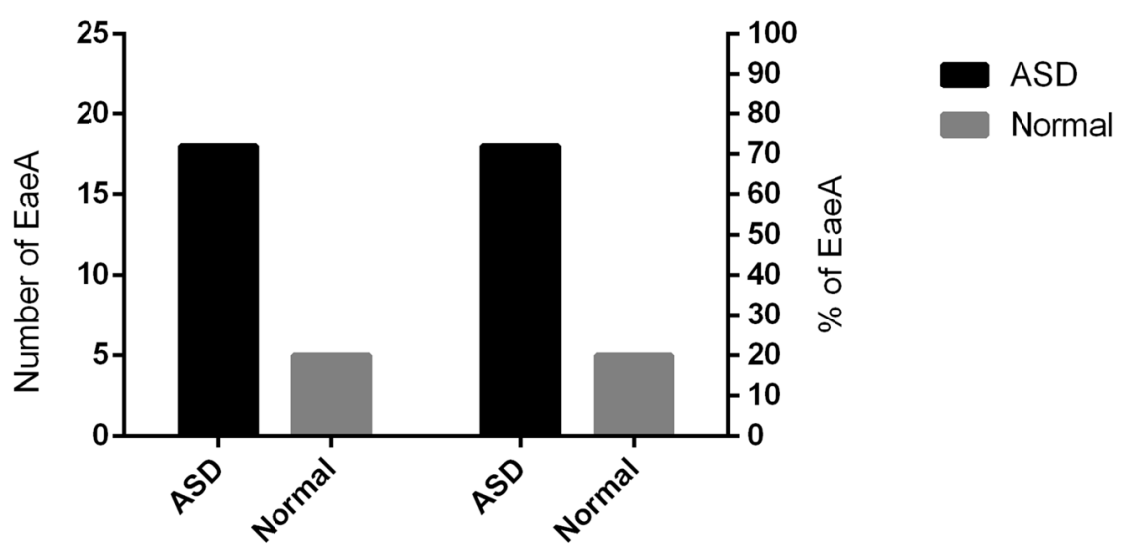

Figure 2. Statistical Comparison of Eae $A$ in Normal and ASD

\subsection{Ligand Preparation, Protein Structure Prediction, Docking}

The modeled structure of intimin (Figure 3) retrieved from Swissmodel workspace had a residue range from 1 to 140 and QMEAN Z-Score: -4.58. The QMEAN4 sicore is a composite score consisting of a linear combination of 4 statistical potential terms (estimated model reliability between $0-1$ ). The pseudo-energies of the contributing terms are given in Table 1 together with their Z-scores with respect to scores obtained for high-resolution experimental structures of similar size solved by X-ray crystallography.

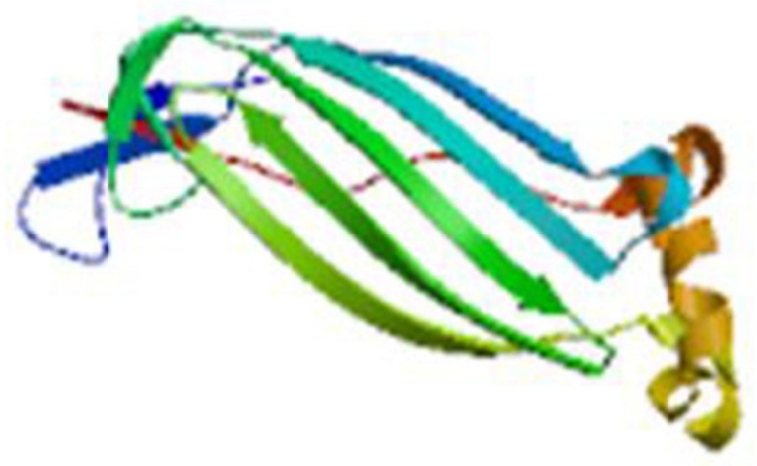

Figure 3. Intimin model structure 


\section{Mll Macrothink}

All the docked conformations gave negative binding energy. Among the three neurotransmitters chosen for the docking study with intimin structure the best i.e minimum binding energy was obtained for serotonin. Docking results are shown in Table 2 in the increasing order of their binding energy. The aminoacids involved in the binding site of intimin are Arginine132, Arginine131, Aspargine 22, Aspargine 134, Isoleucine 135, Isoleucine 136, Tyrosine 14, Alanine 15, Phenylalanine 21, Arginine 24 and Glycine 20. In Figure 4 the ligand is shown as ball-and-stick, surrounded by a molecular surface. The surface is colored with atomic colors in regions that contact the receptor, and gray in regions that are not in contact. Portions of the receptor that are in contact with the ligand are shown with ball-and-stick and mesh work spheres. Hydrogen bonds are shown as a string of small spheres (Morris et al., 2009). The Binding energy was calculated based on the following formula.

Estimated Free Energy of Binding = Final Intermolecular Energy $(\mathrm{vdW}+$ Hbond + desolv Energy + Electrostatic Energy) + Final Total Internal Energy + Torsional Free EnergyUnbound System's Energy where vdW is vanderwaals force interaction enrgy, $\mathrm{H}$ bond is hydrogen bond energy and desolv is desolvation energy (Raschka et al., 2014).

AutoDock gives tools for clustering the results either at the last part of each docking or by combining together multiple docking results and re-clustering them. The single best score resulting fromeach cluster is shown in the output file (Morris et al., 2009). The histogram of cluster analysis of docked conformations of intimin with serotonin and the root mean square deviation (RMSD) values in each cluster of docking were obtained.

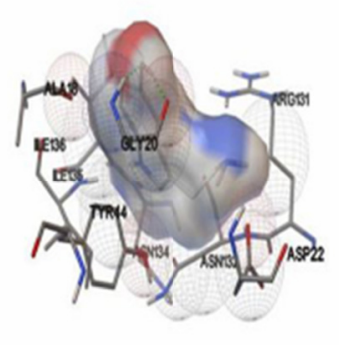

Dockinginteraction of intimin with serotonin

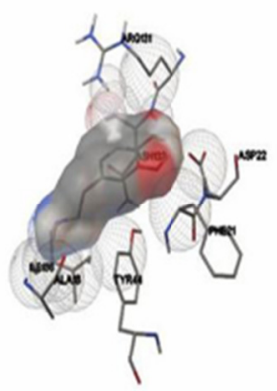

Docking interaction of intimin with acetyl choline

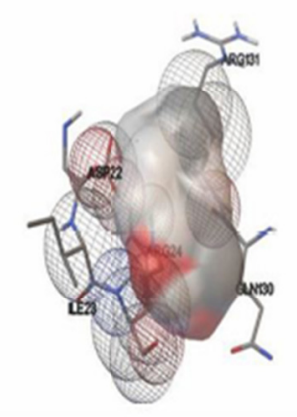

Docking interaction of intimin with dopamine

Figure 4. Docking Interaction of intimin with various selected neurotransmitters 


\section{Discussion}

The medical establishment has often failed to take note of coinciding GI symptoms in autistic children. Downplaying it as a psychiatric disorder, often, physical symptoms common in patients with autism are ignored or unrecognized. It is a known fact that bacterial population in the gut can be beneficial as well pathogenic. Along with causing damage to gut tissue, abnormal bacteria are thought to affect the brain. A gut-brain connection has been hypothesized in autism (Theoharis et al., 2011). The scenario can have its source attributed to the neurotoxic substances derived from abnormal bowel microflora. They can also produce endotoxins that can affect locally and systemically including the brain. When the toxins produced by harmful bacteria are not adequately metabolized, these toxins happen to accumulate in the brain by way of the blood-stream which can lead to delirium, coma and confusion etc which are shown to have in autistic patients in varying degrees.

Intimin is a 97-KDa attachment and effacement protein encoded by eae A (Gafan et al., 2005). The reference pathway of Apoptosis and Regulation of Actin cytoskeleton by KEGG explains how pathogenic Escherichia coli infection can adversely affect the bearer through the presence of intimin causing apoptosis through mitochondrial dysfunction and also by playing a major role in regulation of actin cytoskeleton. We need to investigate if the higher prevalence of EHEC, reported for the first time here, in autistic children in comparison with normal children leads to the above mentioned situations like mitochondrial dysfunction and apoptosis in them which cannot be out rightly rejected.

Out of the three neurotransmitters selected for the in silico study, serotonin is the most affected by the interaction with intimin followed by acetylcholine and dopamine. Serotonin, a monoamine neurotransmitter, primarily found in the Gastrointestinal tract does strongly influence mood and social behavior, appetite, digestion, sleep, memory and sexual desire. It would not be out of place to think that EHEC can affect serotonin metabolism, thereby bringing about the above said features in autism as corroborated in the in silico study.

It is known that enteric infections can cause anxiety, depression and cognitive dysfunction. The neurotransmitters being part and parcel of Central Nervous System and cognitive function, the in silico study we carried which shows minimum binding energy with the selected neurotransmitters unwittingly suggest that altered gut microflora or presence of pathogenic microflora can have substantial influence in cognitive disorders and need to elucidate using wet lab studies.

Having seen that harmful organism like EHEC is a frequent inhabitant in the bowels of autistic children, we need to rid of these harmful bacteria and changing the diet can be a fruitful alternative.

\section{Acknowledgements}

We acknowledge the guidance and support rendered by Dr Nina Shilan MD, FAAP. Also we record our sincere thanks to Rev. Sr. Serena, Rev. Sr. Helen, Rev. Sr. Pushpa and Ms. Sruthi for extending their help in sample collection through the wards under their care at various special schools in Kerala. 


\section{References}

Altschul, S. F., Gish, W., Miller, W., Myers, E. W., \& Lipman, D. J. (1990). Basic local alignment search tool. J. Mol. Biol, 215, 403-410. http://dx.doi.org/10.1016/S0022-2836(05)80360-2

Arnold, K., Bordoli, L., Kopp, J., \& Schwede, T. (2006). The SWISS-MODEL workspace: a web-based environment for protein structure homology modelling. Bioinforma. Oxf. Engl, 22, 195-201. http://dx.doi.org/10.1093/bioinformatics/bti770

Benach, J. L., Li, E., \& McGovern, M. M. (2012). A microbial association with autism. mBio, 3. http://dx.doi.org/10.1128/mBio.00019-12

Blanco, M., Blanco, J. E., Dahbi, G., Mora, A., Alonso, M. P., Varela, G., ... Blanco, J. (2006). Typing of intimin (eae) genes from enteropathogenic Escherichia coli (EPEC) isolated from children with diarrhoea in Montevideo, Uruguay: identification of two novel intimin variants (muB and xiR/beta2B). J. Med. Microbiol, 55, 1165-1174. http://dx.doi.org/10.1099/jmm.0.46518-0

Jepson, B., \& Johnson, J. (2007). Changing the Course of Autism, n.d. Sentient Publ.

Cookson, A. L., \& Woodward, M. J. (2003). The role of intimin in the adherence of enterohaemorrhagic Escherichia coli (EHEC) O157: H7 to HEp-2 tissue culture cells and to bovine gut explant tissues. Int. J. Med. Microbiol. IJMM, 292, 547-553. http://dx.doi.org/10.1078/1438-4221-00235

D’Souza, Y., Fombonne, E., \& Ward, B. J. (2006). No evidence of persisting measles virus in peripheral blood mononuclear cells from children with autism spectrum disorder. Pediatrics 118, 1664-1675. http://dx.doi.org/10.1542/peds.2006-1262

Fagan, P. K., Hornitzky, M. A., Bettelheim, K. A., \& Djordjevic, S. P. (1999). Detection of Shiga-Like Toxin (stx1 and stx2), Intimin (eaeA), and Enterohemorrhagic Escherichia coli (EHEC) Hemolysin (EHEC hlyA) Genes in Animal Feces by Multiplex PCR. Appl. Environ. Microbiol, 65, 868-872.

Finegold, S. M. Dowd, S. E., Gontcharova, V., Liu, C., Henley, K. E., Wolcott, R. D., ... Green III, J. A. (2010). Pyrosequencing study of fecalmicroflora of autistic and control children. Anaerobe, 16, 444-453. http://dx.doi.org/10.1016/j.anaerobe.2010.06.008

Gafan, G. P., Lucas, V. S., Roberts, G. J., Petrie, A., Wilson, M., \& Spratt, D. A. (2005). Statistical analyses of complex denaturing gradient gel electrophoresis profiles. J. Clin. Microbiol, 43, 3971-3978. http://dx.doi.org/10.1128/JCM.43.8.3971-3978.2005

Garrett, M., Morris, D. S. G. n.d Automated Docking of Flexible Ligands to Flexible Receptors.

Jerse, A. E., Yu, J., Tall, B. D., \& Kaper, J. B. (1990). A genetic locus of enteropathogenic Escherichia coli necessary for the production of attaching and effacing lesions on tissue culture cells. Proc. Natl. Acad. Sci. U. S. A. 87, 7839-7843. http://dx.doi.org/10.1073/pnas.87.20.7839

McKee, M. L., \& O’Brien, A. D. (1996). Truncated enterohemorrhagic Escherichia coli (EHEC) O157:H7 intimin (EaeA) fusion proteins promote adherence of EHEC strains to HEp-2 cells. Infect. Immun, 64, 2225-2233. 
Morris, G. M., Huey, R., Lindstrom, W., Sanner, M. F., Belew, R. K., Goodsell, D. S., \& Olson, A. J. (2009). AutoDock4 and AutoDockTools4: Automated docking with selective receptor flexibility. J. Comput. Chem, 30, 2785-2791. http://dx.doi.org/10.1002/jcc.21256

Mundy, R., Schüller, S., Girard, F., Fairbrother, J. M., Phillips, A. D., \& Frankel, G. (2007). Functional studies of intimin in vivo and ex vivo: implications for host specificity and tissue tropism. Microbiol. Read. Engl, 153, 959-967. http://dx.doi.org/10.1099/mic.0.2006/003467-0

Nataro, J. P., \& Kaper, J. B. (1998). Diarrheagenic Escherichia coli. Clin. Microbiol. Rev, 11, 142-201.

Nguyen, Y., \& Sperandio, V. (2012). Enterohemorrhagic E. coli (EHEC) pathogenesis. Front. Cell. Infect. Microbiol, 2. http://dx.doi.org/10.3389/fcimb.2012.00090

Raschka, S. (2014). Molecular docking, estimating free energies of binding, and AutoDock\&\#39's semi-empirical force field.

Theoharides, T. C., \& Zhang, B. (2011). Neuro-inflammation, blood-brain barrier, seizures and autism. J. Neuroinflammation, 8, 168. http://dx.doi.org/10.1186/1742-2094-8-168

Valicenti-McDermott, M., McVicar, K., Rapin, I., Wershil, B. K., Cohen, H., \& Shinnar, S. (2006). Frequency of gastrointestinal symptoms in children with autistic spectrum disorders and association with family history of autoimmune disease. J. Dev. Behav. Pediatr. JDBP 27, S128-136. http://dx.doi.org/10.1097/00004703-200604002-00011

Wang, R. F., Cao, W. W., \& Cerniglia, C. E. (1996). PCR detection and quantitation of predominant anaerobic bacteria in human and animal fecal samples. Appl. Environ. Microbiol, $62,1242-1247$.

Williams, B. L., Hornig, M., Parekh, T., \& Lipkin, W. I., (2012). Application of novel PCR-based methods for detection, quantitation, and phylogenetic characterization of Sutterella species in intestinal biopsy samples from children with autism and gastrointestinal disturbances. mBio 3 .

ACDLabs Freeware, 11, Advanced Chemistry Development, Inc., Toronto, On, Canada, www.acdlabs.com, 2015.

Expasy Bioinformatics Resource portal, expasy.org/translate/

iCon: Molecular file format converter, pipe@ibibiosolutions.com.

Nicolas, G., Alexandre, D., \& Torsten, S. (2006). DeepView/Swiss-PdbViewer Version 3.7 (SP5).

\section{Copyright Disclaimer}

Copyright reserved by the author(s).

This article is an open-access article distributed under the terms and conditions of the Creative Commons Attribution license (http://creativecommons.org/licenses/by/3.0/). 